\title{
JOINT SPATIAL AND TEMPORAL DELTA-SIGMA MODULATION FOR WIDEBAND ANTENNA ARRAYS AND VIDEO HALFTONING
}

\author{
$\begin{array}{lc}\text { Dan P. Scholnik } & \text { Jeffrey O. Coleman } \\ \text { scholnik@nrl.navy.mil } & \text { jeffc@alum.mit.edu }\end{array}$ \\ Naval Research Laboratory \\ Washington, DC
}

\begin{abstract}
Extending an existing architecture for delta-sigma conversion of vector inputs, we suggest spectrally shaping quantization noise jointly in temporal and spatial frequency domains with delta-sigma modulation, and we examine the application of the idea to wideband antenna or acoustic arrays and to halftoning of video imagery.
\end{abstract}

\section{INTRODUCTION}

Delta-sigma $(\Delta \Sigma)$ modulation is widely used in $\mathrm{D} / \mathrm{A}$ and $\mathrm{A} / \mathrm{D}$ conversion to obtain high precision, linearity, and dynamic range in exchange for a higher sampling rate or lower bandwidth [I] by placing a low-resolution quantizer inside a feedback loop to spectrally shape quantization noise to minimize its interference with the signal. Out-of-band quantization noise is then removed with digital or analog filtering according to the application (A/D or D/A). High oversampling rates (rates relative to signal bandwidths) result in signal-to-noise ratio (SNR) levels often exceeding the roughly $100 \mathrm{~dB}$ limit of conventional (Nyquist-rate) converters. One-bit quantizers have traditionally been used for their automatically near-ideal characteristics, but newer multi-bit $\Delta \Sigma$ like architectures [2, 3, 4, 5] spectrally shape hardware-mismatch errors as well and lower oversampling requirements by increasing parallelism.

Spatial $\Delta \Sigma$ modulation of images to produce halftone images from continuous-tone ones is known as error diffusion [6, 7]. Typically, continuous-valued image files viewed on a monitor at 100 dots per inch (DPI) are converted to 600+ DPI binary-valued images for printing. Single-bit quantizers are used, but less for being ideal than because they model printers and displays with binaryvalued pixels. The inherent lowpass nature of human vision then replaces the explicit post-modulator filtering that removes quantization noise in temporal $\Delta \Sigma$ modulation. The eye's sensitivity is much lower than the $100 \mathrm{~dB}$ mentioned before, so lower oversampling rates and filter orders are typically used in halftoning than in temporal applications. Further, human vision is necessarily subjective, and so SNR measures can involve more than just in-band signal and noise power levels.

Given the benefits of temporal and spatial $\Delta \Sigma$ modulators individually, it is natural to consider jointly oversampling space and time in a space-time $\Delta \Sigma$ modulator. An obvious application is halftoned video sequences for simple binary displays. A perhaps more technically challenging application is digitally controlled transmit arrays of medium to high bandwidth for RF (communications, radar) or acoustic (sonar) systems in which hardware simplicity and perfect amplifier linearity make single-bit outputs attractive. Here we first extend previous $\Delta \Sigma$ results to the case of joint space-time modulation and then consider the applications.

This work was supported by the Office of Naval Research

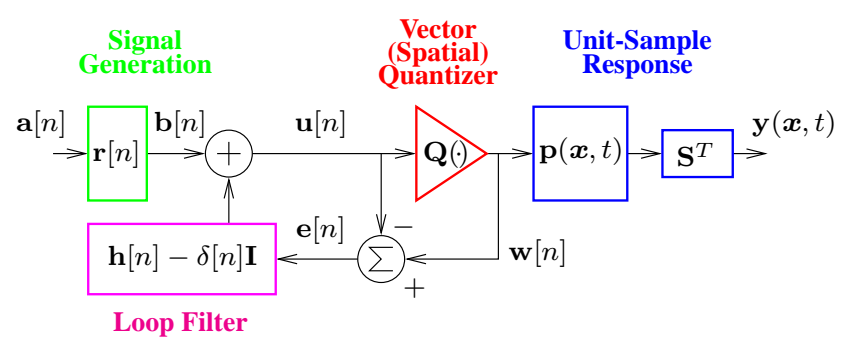

Fig. 1. Space-time $\Delta \Sigma$ modulator. The loop filter matrix is square. The shapes of other boxes indicate relative matrix dimensions.

\section{SPACE-TIME $\Delta \Sigma$ MODULATION}

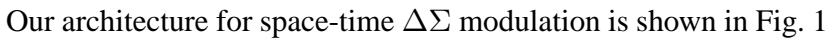
and is a natural extension of our earlier vector $\Delta \Sigma$ architecture [8]. Input vector $\mathbf{a}[n]$ represents the original, high-resolution signal to be $\Delta \Sigma$-modulated, and the Signal Generation block applies unit-sample response matrix $\mathbf{r}[n]$ to create spatially and temporally bandlimited input $\mathbf{b}[n]$ for input to the $\Delta \Sigma$ modulation loop. In a wideband array, $N \times 1$ vector a $[n]$ might contain scalar waveforms to be transmitted, and columns of $M \times N$ matrix $\mathbf{r}[n]$ would act as individual beamformers to generate individual array-element drives. For video, $K N \times 1$ vector sequence $\mathbf{a}[n]$ of two-dimensional images with $N$ pixels of $K$ color components each could be spatially and temporally interpolated using $M \times K N$ matrix $\mathbf{r}[n]$.

Each vector coordinate inside the $\Delta \Sigma$ loop corresponds to a hardware unit element, perhaps a switch feeding a radiator in an array or a color component (i.e. RGB) of a pixel. The loop is closed around a Loop Filter operating on the quantization error of a Vector (Spatial) Quantizer. Sequential computability requires strict causality in the loop-filter impulse response, or $\mathbf{h}[0]=\mathbf{I}$. The quantizer output is the $\Delta \Sigma$-modulated space-time signal.

Matrix Unit-Sample Response $\mathbf{p}(\boldsymbol{x}, t)$ and matrix $\mathbf{S}^{T}$ characterize the physics of combining unit-element outputs at time $t$ after a unit-sample input at position $\boldsymbol{x}$. The mathematically unnecessary decomposition into two matrices is convenient for hardware modeling. For arrays, $\mathbf{S}^{T} \mathbf{p}(\boldsymbol{x}, t)$ is a $L \times M$ matrix, with $L$ the number of electromagnetic polarization components or acoustic propagation modes of interest and $M$ the number of unit elements. For $K$ color-component video imagery, it is a $K \times M$ matrix.

Begin the detailed analysis of Fig. Twith the quantizer input,

$$
\mathbf{u}[n]=(\mathbf{r} * \mathbf{a})[n]+(\mathbf{h} * \mathbf{e})[n]-\mathbf{e}[n],
$$

where $*$ represents conventional discrete-time matrix-vector convolution. The quantizer output becomes

$$
\mathbf{w}[n]=(\mathbf{r} * \mathbf{a})[n]+(\mathbf{h} * \mathbf{e})[n] .
$$


Defining a temporal convolution in continuous/discrete-time by

$$
(\mathbf{p} \star \mathbf{b})(\boldsymbol{x}, t) \triangleq T \sum_{k} \mathbf{p}(\boldsymbol{x}, t-k T) \mathbf{b}[k],
$$

with $T$ denoting both the system sampling interval and transpose, ${ }^{T}$ the final system output, before/after Fourier transforming on $t$, is

$$
\begin{aligned}
\mathbf{y}(\boldsymbol{x}, t)= & \left(\mathbf{S}^{T} \mathbf{p} \star \mathbf{r} * \mathbf{a}\right)(\boldsymbol{x}, t)+ \\
& \left(\mathbf{S}^{T} \mathbf{p} \star \mathbf{h} * \mathbf{e}\right)(\boldsymbol{x}, t), \\
\mathbf{Y}(\boldsymbol{x}, f)= & \mathbf{S}^{T} \mathbf{P}(\boldsymbol{x}, f) T \mathbf{R}(f T) \mathbf{A}(f T)+ \\
& \mathbf{S}^{T} \mathbf{P}(\boldsymbol{x}, f) T \mathbf{H}(f T) \mathbf{E}(f T) .
\end{aligned}
$$

On the right in each the first and second terms are the desired signal and the quantization error respectively, each shaped by the pulse matrix after filtering. To suppress the error term at spatio-temporal frequencies containing desired signal components, one must carefully choose the spatial locations of unit elements (antenna elements, transducers, or ink dots), the vector-quantization rule, the signal and noise frequency responses $\mathbf{R}(f T)$ and $\mathbf{H}(f T)$, and possibly even pulse-response matrix $\mathbf{p}(\boldsymbol{x}, t)$. We next consider the details for arrays and video sequences separately.

\section{APPLICATIONS}

\subsection{Wideband Antenna Arrays}

A new generation of RF transmitters keeps signals digital nearly to the individual antenna-element inputs where D/A conversion, ana$\log$ filtering, and power amplication finally takes place. Tremendous flexibility in choosing array patterns results [9, 10], ideally for each of several signals separately. Intermodulation will be unacceptable, however, unless any high-power amplifiers handling multiple simultaneous signals are extraordinarily linear. One option is to forego conventional amplifiers and instead $\Delta \Sigma$ modulate high-power switches [11]. Adding closely spaced antenna elements to this scheme boosts output power per unit aperture and opens the door to joint spatial and temporal $\Delta \Sigma$ modulation. []

To analyze such a system, let element $k$ of vector $\mathbf{w}[n]$ in Fig. 1 be the time $n T$ input to high-power switch $k$ feeding an antenna element at position $\mathbf{x}_{k}$. Let the $k$ th diagonal $3 \times 1$ block of $\mathbf{p}(\boldsymbol{x}, t)$ model linear, time-invariant propagation from switch input $k$ to the fields at position $\boldsymbol{x}$ so that elements $3 k, 3 k+1$, and $3 k+2$ of vector $(\mathbf{p} \star \mathbf{w})(\boldsymbol{x}, t)$ are the $x, y$, and $z$ components of the electric field at position $\boldsymbol{x}$ and time $t$. Off-block-diagonal entries represent crosstalk and mutual coupling between elements. Superposition of switch/element fields is modeled by block matrix $\mathbf{S}^{T}=\left[\mathbf{I}_{3}, \mathbf{I}_{3}, \ldots, \mathbf{I}_{3}\right]$, assembled from $3 \times 3$ identity matrices. System output $\mathbf{y}(\boldsymbol{x}, t)$ becomes a $3 \times 1$ vector of field components.

\section{Array Pattern and Noise Transfer Function}

We now examine in more detail the responses that modify signal and noise in (2). Writing the $k$ th column of $\mathbf{s}^{T} \mathbf{P}(\boldsymbol{x}, f)$ as $\mathbf{P}_{k}(\boldsymbol{x}-$

\footnotetext{
${ }^{1}$ The situation is similar for ultrasonic arrays; see [12].

${ }^{2}$ Summing multiple switch outputs together to feed a single physical element would allow shaping of hardware-mismatch errors using dynamic element matching (DEM) [8, 5]. This is simply modeled with nonunique $x_{k}$ 's. A DEM version of this system would require generalization of our analysis however, which for simplicity assumes that $k \mapsto x_{k}$ is one to one.
}

$\left.\mathbf{x}_{k}, f\right)$, the desired-signal (first) term of (2) becomes

$$
\begin{aligned}
& \mathbf{Y}_{s}(\boldsymbol{x}, f)=\sum_{k} \mathbf{P}_{k}\left(\boldsymbol{x}-\mathbf{x}_{k}, f\right) T \sum_{n} R_{k, n}(f T) A_{n}(f T) \\
& \hat{\mathbf{Y}}_{s}(\boldsymbol{v}, f)=\sum_{k} \hat{\mathbf{P}}_{k}(\boldsymbol{v}, f) T \sum_{n} R_{k, n}(f T) e^{-j 2 \pi \mathbf{x}_{k}^{T} \boldsymbol{v}} A_{n}(f T),
\end{aligned}
$$

where we have transformed to the far field (see Appendix A]) in the second line. If element responses are identical then $\hat{\mathbf{P}}_{k}(\boldsymbol{v}, f)=$ $\hat{\mathbf{P}}_{0}(\boldsymbol{v}, f)$ can be factored from the summation over $k$, which is then recognized as a spatial Fourier transform:

$$
\hat{\mathbf{Y}}_{s}(\boldsymbol{v}, f)=\hat{\mathbf{P}}_{0}(\boldsymbol{v}, f) T \sum_{n} \hat{R}_{n}(\boldsymbol{v}, f T) A_{n}(f T) .
$$

This extends the classic array-pattern formulation to multiple inputs, with the pattern seen by $n$th input $A_{n}(f T)$ just the product of element pattern $\hat{\mathbf{P}}_{0}(\boldsymbol{v}, f)$ and the $n$th array factor $T \hat{R}_{n}(\boldsymbol{v}, f T)$.

Taking the error term of (2) through similar stages,

$$
\begin{aligned}
\mathbf{Y}_{e}(\boldsymbol{x}, f) & =\sum_{k} \mathbf{P}_{k}\left(\boldsymbol{x}-\mathbf{x}_{k}, f\right) T \sum_{n} H_{k n}(f T) E_{n}(f T) \\
\hat{\mathbf{Y}}_{e}(\boldsymbol{v}, f) & =\hat{\mathbf{P}}_{0}(\boldsymbol{v}, f) T \sum_{k, n} H_{k, n}(f T) e^{-j 2 \pi \mathbf{x}_{k}^{T} \boldsymbol{v}} E_{n}(f T) \\
& =\hat{\mathbf{P}}_{0}(\boldsymbol{v}, f) T \sum_{n} \hat{H}_{n}(\boldsymbol{v}, f T) E_{n}(f T) .
\end{aligned}
$$

Frequency repsonse $\hat{H}_{n}(\boldsymbol{v}, f T)$ is the spatial and temporal shaping of element $E_{n}(f T)$ of the quantization error.

How does (5) relate to the spatio-temporal spectrum of the quantization error? Suppose element locations $\left\{\mathbf{x}_{k}\right\}$ are distinct and that interpolation function $\psi_{n}(\boldsymbol{x})$, with Fourier transform $\Psi_{n}(\boldsymbol{v})$, is constructed such that $\psi_{n}\left(-\boldsymbol{x}_{m}\right)=\delta_{n m}$, a Kronecker delta. (The minus sign will prove convenient.) Insert

$$
\sum_{m} \int \psi_{n}(-\boldsymbol{x}) \delta\left(\boldsymbol{x}-\mathbf{x}_{m}\right) d \boldsymbol{x}=\sum_{m} \delta_{n m}=1
$$

and two superfluous conjugations into (4) and rearrange:

$$
\begin{aligned}
\hat{\mathbf{Y}}_{e}(\boldsymbol{v}, f)= & \\
\hat{\mathbf{P}}_{0}(\boldsymbol{v}, f) & \int\left(T\left[\sum_{n, k} e^{-j 2 \pi \mathbf{x}_{k}^{T} \boldsymbol{v}} H_{k, n}(f T) \psi_{n}(-\boldsymbol{x})\right]^{*}\right)^{*} \\
& \times\left(\sum_{m} E_{m}(f T) \delta\left(\boldsymbol{x}-\mathbf{x}_{m}\right)\right) d \boldsymbol{x} .
\end{aligned}
$$

Parseval's relation applies. Fourier transforming parenthesized quantities from spatial variable $\boldsymbol{x}$ to a spatial-frequency variable, say $\boldsymbol{\eta}$,

$$
\begin{aligned}
\hat{E}(\boldsymbol{\eta}, f T) & \triangleq \sum_{m} E_{m}(f T) e^{-j 2 \pi \boldsymbol{x}_{m}^{T} \boldsymbol{\eta}} \\
\hat{H}^{*}(\boldsymbol{v}, \boldsymbol{\eta} ; f T) & \triangleq T \sum_{n, k}\left[e^{-j 2 \pi \boldsymbol{x}_{k}^{T} \boldsymbol{v}} H_{k, n}(f T)\right]^{*} \Psi_{n}^{*}(\boldsymbol{\eta}) .
\end{aligned}
$$

Parseval's relation now reveals (6) to be a linear-system integral:

$$
\hat{\mathbf{Y}}_{e}(\boldsymbol{v}, f)=\hat{\mathbf{P}}_{0}(\boldsymbol{v}, f) \int \hat{H}(\boldsymbol{v}, \boldsymbol{\eta} ; f T) \hat{E}(\boldsymbol{\eta}, f T) d \boldsymbol{\eta} .
$$

Linear-system input-output relation (9) is the heart of the matter. The final parenthesized quantity in (6) is the error signal concentrated spatially on element locations $\left\{\mathbf{x}_{k}\right\}$, here acted on in the 
transform domain in (9) by noise-shaping response $\hat{H}(\boldsymbol{v}, \eta ; f T)$. This depends on one temporal input-output frequency but on input and output spatial frequencies separately. It therefore represents a linear system that is time-invariant but not space invariant [13]. Its response should be designed to distribute the noise in its output space $\mathbb{R}^{3} \times \mathbb{R}$ of spatio-temporal frequencies $(\boldsymbol{v}, f)$ so that the final application in (9) of $\hat{P}(\boldsymbol{v}, f)$, the spatio-temporal frequency response of the antenna element, will discard most of the noise. Identical elements have made this element system space invariant as well as time invariant. Further, its spatio-temporal frequency response is automatically zero except on the Helmholtz cone defined by $|f|=\|v\|$, the "visible region" of spatio-temporal frequency space (Appendix A).

\section{Spatial and Temporal Oversampling}

Strict-causality requirement $\mathbf{h}[0]=\mathbf{I}$ prohibits suppressing quantization noise everywhere, so the noise must instead be placed out of band. Where is that? Element locations $\left\{\mathbf{x}_{k}\right\}$ and sampling interval $T$ control the structure of the signal and noise input to the element system, and both are well defined and nonzero on the whole $(\boldsymbol{v}, f)$ space. Period $T^{-1}$ in temporal frequency $f$ has been implied throughout by the discrete-time system structure. Arbitrary element locations can lead to aperiodic functions of spatial frequency, but if the elements lie on some lattice $\Lambda \mathbb{Z}^{3}$ defined by matrix $\Lambda$ then both signal and noise terms are periodic in spatial frequency as well with period $\boldsymbol{\Lambda}^{-T}$ Cartesian product $\boldsymbol{\Lambda}^{-T} \times T^{-1}$ then gives the overall spatio-temporal frequency period. This must be made large enough to include an out-of-band region, preferably off the visible (Helmholtz) cone entirely. Once this is done, filter responses $\hat{R}_{n}(\boldsymbol{v}, f T)$ and $\hat{H}(\boldsymbol{v}, \boldsymbol{\eta} ; f T)$ can be chosen to maximize SNR in the in-band portion of the visible region.

As a simple example, take elements uniformly spaced at interval $d$ along the $x$ axis, and use spatial frequency $\boldsymbol{v}=\left(v_{x}, v_{y}, v_{z}\right)^{T}$. Rewriting the right-hand sides of (3) and (5) as $\hat{\mathbf{P}}_{0}(\boldsymbol{v}, f) \hat{W}_{s}(\boldsymbol{v}, f)$ and $\hat{\mathbf{P}}_{0}(\boldsymbol{v}, f) \hat{W}_{e}(\boldsymbol{v}, f)$, the implicitly defined signal and noise spectra $\hat{W}_{s}(\boldsymbol{v}, f)$ and $\hat{W}_{e}(\boldsymbol{v}, f)$ have period $1 / d$ in $v_{x}$ and are unchanging as $v_{y}$ and $v_{z}$ vary. Projecting into the $\left(v_{x}, f\right)$ plane and considering only positive temporal frequencies, we have the four choices illustrated in Fig. \&. The central trapezoid is the in-band portion of the visible cone, projected into the plane, and the bounding rectangle is one period of the signal and noise spectra and corresponds to particular choices of $T$ and $d$. Out-of-band regions where quantization noise can be placed are shaded. In the upper right, $T^{-1}$ is chosen just slightly larger than the signal bandwidth, and $d=c / 2 f_{h}=\lambda_{h} / 2$, the classic choice. There is no oversampling, and no noise shaping is possible. On the bottom right, an increased $T^{-1}$ allows noise shaping in temporal frequency by using independent scalar $\Delta \Sigma$ converters to drive the individual elements of a conventional array (suggested in [14]). On the upper left, there is oversampling in space only, corresponding to running a $\Delta \Sigma$ modulator across the array at each time interval, a sort of instant-by-instant RF halftoning. Joint temporal and spatial oversampling appears at the lower left. The much larger area in which to place noise should allow much better performance.

\subsection{Video Halftoning}

Video halftoning is more straightforward. Signal sequences are temporally lowpass, not bandpass. And at each time instant, images are lowpass functions of two spatial dimensions, not three,

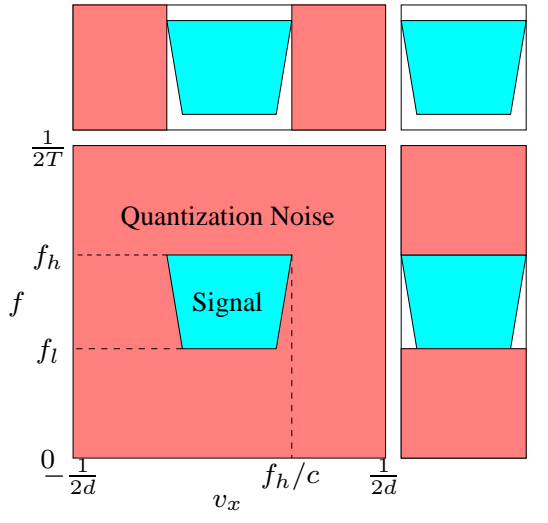

Fig. 2. Example signal and quantization-noise regions.

and the unit elements (pixels) are generally on a rectangular lattice. Moreover, the relevant frequency domain is not based on coherent far-field propagation but instead on the straightforward temporospatial Fourier spectrum of the video intensity distribution. The bandlimited nature of wave propagation (the visible region) is also unimportant. It is the lowpass nature of the eye, both spatially and temporally, that filters out high-frequency quantization errors.

In Fig. 廿, each color gets some consecutive number of elements in each vector, possibly a different number for each color, depending on the relative densities of different-colored pixels and their locations. Composite pixels are simply separate monochrome pixels with identical locations. Spatio-temporally oversampled lowpass signal $\mathbf{b}[n]$ represents spatio-temporal intensity distributions input to the modulator loop. The quantizer output represents the binary intensities driving display pixels. Pixel-intensity response matrix $\mathbf{p}(\boldsymbol{x}, t)$ representing the display is block diagonal by color with each block itself diagonal by pixel location. With $K \times 1$ block-diagonal $\mathbf{S}^{T}=\operatorname{diag}\left(\mathbf{s}^{T}, \ldots, \mathbf{s}^{T}\right)$ and $\mathbf{s}^{T}=(1, \ldots, 1)$, the video output is compactly represented as the $K \times 1$ vector of color components $\mathbf{y}(\boldsymbol{x}, t)$.

\section{SUMMARY AND CONCLUSIONS}

We have presented the basic theory of and an architecture for joint spatial and temporal $\Delta \Sigma$ modulation, focusing on key potential applications. Much work remains to be done in extending existing $\Delta \Sigma$ stability theory and loop filter design to the joint space-time case, as well as in quantizer design. As an example of the latter, the vector quantizer in Fig. 1 might itself be a spatial-only $\Delta \Sigma$ modulator, so that the resulting errors are already shaped spatially.

\section{ACKNOWLEDGMENT}

We wish to thank Dr. Daniel Purdy of the Office of Naval Research and Dr. Mark Rodwell of the University of California, Santa Barbara for reviving our dormant interest in the RF application of this concept, which application they independently discovered and more-thoroughly appreciated, and for strongly encouraging us to push our work forward.

\section{REFERENCES}

[1] J. C. Candy and G. C. Temes, "Oversampling methods for A/D and D/A conversion," in Oversampled Delta-Sigma Data Converters. IEEE Press, New York, 1991. 
[2] R. Schreier and B. Zhang, "Noise-shaped multibit D/A convertor employing unit elements," Electronics Letters, vol. 31, no. 20, pp. 1712-1713, Sept. 1995.

[3] I. Galton, "Spectral shaping of circuit errors in digital-toanalog convertors," IEEE Trans. Circuits and Systems II, vol. 44, no. 10, pp. 808-817, Oct. 1997.

[4] Luis Hernández, "A model of mismatch-shaping D/A conversion for linearized DAC architectures," IEEE Trans. Circuits and Systems I, vol. 45, no. 10, pp. 1068-1076, Oct. 1998.

[5] D. P. Scholnik and J. O. Coleman, "Joint shaping of quantization and hardware-mismatch errors in a multibit delta-sigma DAC," in Proc. 2000 Midwest Symp. on Circuits and Systems (MWSCAS 2000), Lansing MI, Aug. 2000.

[6] R. Floyd and L. Steinberg, "An adaptive algorithm for spatial grayscale," Proc. Soc. Image Display, vol. 17, no. 2, pp. 7577, 1976.

[7] T. D. Kite, B. L. Evans, A. C. Bovik, and T. L. Sculley, "Digital halftoning as 2-D delta-sigma modulation," in Proc. IEEE Int. Conf. Image Processing, 1997, pp. 799-802.

[8] D. P. Scholnik and J. O. Coleman, "Vector delta-sigma modulation with integral shaping of hardware-mismatch errors," in IEEE 2000 Int'l Symp. on Circuits and Systems (ISCAS 2000), Geneva, Switzerland, May 2000.

[9] D. P. Scholnik and J. O. Coleman, "Optimal design of wideband array patterns," in Proc. IEEE Int'l Radar Conference (RADAR 2000), Alexandria VA, May 2000.

[10] D. P. Scholnik and J. O. Coleman, "Formulating wideband array-pattern optimizations," in Proc. IEEE Int'l Symp. Phased Array Systems and Technology (ISPAST 2000), Dana Point, CA, May 2000.

[11] Arun Jayaraman, P. F. Chen, G. Hanington, L. Larson, and P. Asbeck, "Linear high-efficiency microwave power amplifiers using bandpass delta-sigma modulators," IEEE Microwave and Guided Wave Letters, vol. 8, no. 3, Mar. 1998.

[12] Y. Tamura, N. Kawakami, O. Akasaka, M. Okada, and K. Koyama, "Beam-forming using multidimensional sigmadelta modulation," in Proc. IEEE Ultrasonics Symp., 1998, pp. 1077-1080.

[13] Jeffrey O. Coleman, "The fundamental input/output structure of a linear, time-varying array receiver," in Proc. IEEE Int. Conf. Acoustic, Speech, and Signal Processing, Salt Lake City, UT, May 2001, Submitted.

[14] G. Fischer, "Delay-sum beamforming using delta-sigma modulated inputs," in Proc. 2000 Midwest Symp. on Circuits and Systems, Lansing MI, USA, Aug. 2000.

[15] B. E. A. Saleh and M. C. Teich, Fundamentals of Photonics, Wiley Series in Pure and Applied Optics. John Wiley \& Sons, Inc., 1991.

\section{APPENDIX A. THE FAR-FIELD ANTENNA PATTERN}

Here we review the far-field spatial response of an isotropic radiator located at $\mathbf{x}_{0}$, relating it to a four-dimensional function of spatial and temporal frequency. The spatial and temporal response

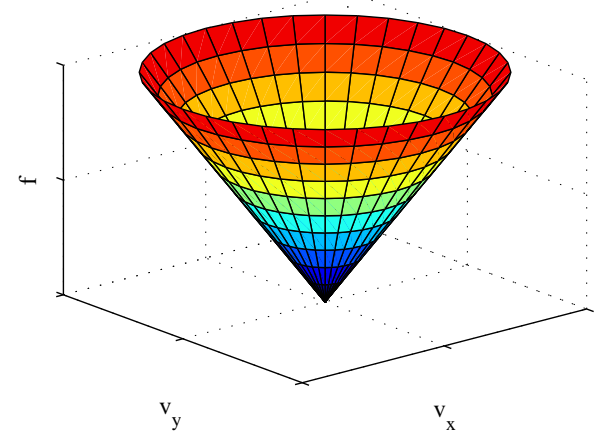

Fig. 3. The visible cone in two spatial-frequency dimensions.

(i.e. the electric field component of interest) of a spatially-offset isotropic antenna element to a (temporal) input impulse is

$$
p\left(\boldsymbol{x}-\mathbf{x}_{0}, t\right)=\frac{1}{\left\|\boldsymbol{x}-\mathbf{x}_{0}\right\|} \delta\left(t-\left\|\boldsymbol{x}-\mathbf{x}_{0}\right\| / c\right) .
$$

By the physics of electromagnetic propagation, the field is causal in time, propagates at a speed $c$, and has field strength decaying as reciprocal distance. The norms in (IID) expand to

$$
\left\|\boldsymbol{x}-\mathbf{x}_{0}\right\|=\|\boldsymbol{x}\| \sqrt{1-\frac{2 \mathbf{x}_{0}^{T} \boldsymbol{x}}{\|\boldsymbol{x}\|^{2}}+\frac{\left\|\mathbf{x}_{0}\right\|^{2}}{\|\boldsymbol{x}\|^{2}}} .
$$

In the far field defined as $\|\boldsymbol{x}\| \gg\left\|\mathbf{x}_{o}\right\|$, the square root is of form $\sqrt{1-2 \delta} \approx 1-\delta$, so $(10)$ with a change of variable becomes

$$
\|\boldsymbol{x}\| p\left(\boldsymbol{x}-\mathbf{x}_{0}, t+\|\boldsymbol{x}\| / c\right) \approx \delta\left(t+\frac{\mathbf{x}_{0}^{T} \boldsymbol{x}}{c\|\boldsymbol{x}\|}-\frac{\left\|\mathbf{x}_{0}\right\|^{2}}{2 c\|\boldsymbol{x}\|}\right) .
$$

Fourier transforming on $t$ results in

$$
\|\boldsymbol{x}\| P\left(\boldsymbol{x}-\mathbf{x}_{0}, f\right) e^{j 2 \pi\|\boldsymbol{x}\| f / c} \approx e^{j 2 \pi \frac{\mathbf{x}_{0}^{T} \boldsymbol{x}}{\|\boldsymbol{x}\| c} f} e^{-j \pi \frac{\left\|\mathbf{x}_{0}\right\|^{2}}{\|\boldsymbol{x}\| c} f} .
$$

If $|f|<f_{0}$ for all frequencies of interest, $\left\|\mathbf{x}_{0}\right\|<D$ for all potential offsets $\mathbf{x}_{0}$, and $D^{2} f_{0} / c \ll\|\boldsymbol{x}\|$, then the second exponential is approximately unity and the right side of (II) becomes

$$
P_{\mathbf{x}_{0}}(\boldsymbol{u}, f) \triangleq e^{j 2 \pi \mathbf{x}_{0}^{T} \boldsymbol{u} f / c}
$$

using unit direction vector $\boldsymbol{u}=\boldsymbol{x} /\|\boldsymbol{x}\|$. This is the Fraunhofer approximation [15, p. 123] where the "output plane" is here the single far-field point $\boldsymbol{x}$. Propagation to the Fraunhofer region effectively Fourier transforms the field spatially. Substitution of spatial frequency $\boldsymbol{v}=-\boldsymbol{u} f / c$ (reciprocal-distance units) yields

$$
\hat{P}_{\mathbf{x}_{0}}(\boldsymbol{v}) \triangleq e^{-j 2 \pi \mathbf{x}_{0}^{T} \boldsymbol{v}},
$$

the spatial Fourier transform of an impulse at $\mathbf{x}_{0}$, a convenient model of an isotropic radiator. By the definition of $\boldsymbol{v}$ we have the Helmholtz equation $\|\boldsymbol{v}\|=|f| / c$, which restricts the spatial frequencies of propagating waves with temporal frequency $f$ to a spherical surface of radius $|f| / c$. Other spatial-frequency components, so-called evanescent waves, die out a small distance from the antenna. So, far-field propagation is an ideal spatial bandpass filter, or if only a single component of $\boldsymbol{v}$ is considered, a spatial lowpass filter with temporal-frequency-dependant cutoff at $|f| / c$.

Generally, the fields radiated into the far field are given by a four-dimensional spatio-temporal transfer function [13] of form $\hat{P}(\boldsymbol{v}, f)=\hat{P}(\boldsymbol{v}) \delta(f-c\|\boldsymbol{v}\|)$. All of its mass is on the visible cone defined by the Helmholtz equation and shown in Fig. 3 . 